Jan 2001

UM-P-2001/006

\title{
Are mirror worlds opaque?
}

\author{
R. Foot \\ Research Centre for High Energy Physics, \\ School of Physics, \\ University of Melbourne, \\ Victoria 3010 Australia
}

\begin{abstract}
Over the last few years, many close orbiting ( $\sim 0.05$ A.U.) large mass planets $\left(\sim M_{J}\right)$ of nearby stars have been discovered. Their existence has been inferred from tiny Doppler shifts in the light from the star and in one case a transit has been observed. Because ordinary planets are not expected to be able to form this close to ordinary stars due to the high temperatures, it has been speculated that the close-in large planets are in fact exotic heavenly bodies made of mirror matter. We show that the accretion of ordinary matter onto the mirror planet (from e.g.the solar wind from the host star) should make the mirror planet opaque to ordinary radiation with an effective radius $\left(R_{p}\right)$ large enough to explain the measured size of the transiting close-in extrasolar planet, HD209458b. Furthermore we obtain the rough prediction that $R_{p} \propto \sqrt{\frac{T_{s}}{M_{p}}}$ (where $T_{s}$, is the surface temperature of the ordinary matter in the mirror planet and $M_{p}$ is the mass of the mirror planet) which will be tested in the near future as more transiting planets are found. We also show that the mirror world interpretation of the close-in extra solar planets explains the low albedo of $\tau$ Boo b because the large estimated mass of $\tau$ Boo b $\left(\sim 7 M_{J}\right)$ implies a small effective radius of $R_{p} \approx 0.5 R_{J}$ for $\tau$ Boo.
\end{abstract}

*Email address: foot@physics.unimelb.edu.au 
Over the last few years a number of planets orbiting nearby stars have been discovered (for a review and references see [1]). Their existence has been inferred from tiny Doppler shifts in the light from the star due to its orbit around the center of mass. The magnitude and periodicity of the Doppler shifts can be used to determine the mass $\times \operatorname{sinI}$ and orbital radius of the planet (where $I$ is the orbital inclination of the planet). In one case, the planet HD209458b transits its star (which means that $\sin I \simeq 1$ ) which allows an accurate determination of the size and mass of the planet [2].

A surprising characteristic of these planets is that some of them have been found which have orbits very close to their star $(\sim 0.05$ A.U.). The existence of close-in giant planets is surprising because it is thought to be too hot for giant planet formation to occur. In Ref. [3] it was suggested that close-in planets might be naturally explained if they are exotic bodies made of mirror matter (rather than ordinary matter as generally assumed). The existence of mirror matter is motivated from particle physics, since mirror particles are predicted to exist if parity and indeed time reversal are unbroken symmetries of nature [4.5]. The idea is that for each ordinary particle, such as the photon, electron, proton and neutron, there is a corresponding mirror particle, of exactly the same mass as the ordinary particle. For example, the mirror proton and the ordinary proton have exactly the same massin. Furthermore the mirror proton is stable for the same reason that the ordinary proton is stable, and that is, the interactions of the mirror particles conserve a mirror baryon number. The mirror particles are not produced in Laboratory experiments just because they couple very weakly to the ordinary particles. In the modern language of gauge theories, the mirror particles are all singlets under the standard $G \equiv S U(3) \otimes S U(2)_{L} \otimes U(1)_{Y}$ gauge interactions. Instead the mirror particles interact with a set of mirror gauge particles, so that the gauge symmetry of the theory is doubled, i.e. $G \otimes G$ (the ordinary particles are, of course, singlets under the mirror gauge symmetry) [5]. Parity is conserved because the mirror particles experience right-handed mirror weak interactions and the ordinary particles experience the usual left-handed weak interactions. Ordinary and mirror particles interact with each other predominately by gravity onlyf. At the present time there is a range of experimental evidence supporting the existence of mirror matter. Firstly, it provides a natural candidate for dark matter, which might be mirror stars (and mirror dust, planets etc) [10]. There is an interesting possibility that these mirror stars have already been detected experimentally in the MACHO experiments [11]. Secondly, ordinary and mirror neutrinos are maximally mixed with each other if neutrinos have mass [12]. This nicely

$\dagger$ The mass degeneracy of ordinary and mirror matter is only valid provided that the parity symmetry is unbroken, which is the simplest and theoretically most attractive possibility. For some other possibilities, which invoke a mirror sector where parity is broken spontaneously (rather than being unbroken), see Ref. [6].

${ }^{\ddagger}$ It is possible to have small non gravitational interactions between ordinary and mirror matter. Assuming gauge invariance and renormalizability the only possibilities are photon -mirror photon kinetic mixing [7,8,5] and Higgs - mirror Higgs mixing [5,9]. 
explains the solar and atmospheric neutrino anomaliess. The idea is also compatible with the LSND experiment [12]. Interestingly, maximal ordinary - mirror neutrino oscillations do not pose any problems for big bang nucleosynthesis (BBN) and can even fit the inferred primordial abundances better than the standard model [15]. Finally there is also tantalizing experimental evidence of the mirror world from the orthopositronium lifetime anomaly which can be explained [16] due to the effects of photon-mirror photon kinetic mixing [7].

Because mirror matter interacts predominately by gravity only, it is not heated up by the ordinary photons emitted by the host star. Thus, any mirror matter present in a stellar nebula can form close to the host star without any apparent theoretical problems. In fact such a possibility was effectively predicted by Blinnikov and Khlopov in 1982 [17] where they discussed the possibility of having a close-in mirror planet with an orbit inside the radius of the sun.

Interestingly, the "dynamical" mirror image system of a mirror star with an ordinary planet would appear to ordinary observers like us as an isolated ordinary planet. Thus, the recent discovery [18] of isolated planets is not particularly surprising from this perspective [19]. For a close-in ordinary planet the periodic Doppler shift in the frequencies should be of order $10^{-3}$ providing a simple test of this idea [19].

At first sight, one might think that a mirror planet would be transparent to ordinary radiation with no scattered or reflected light (i.e. its albedo would be zero). This would be true of a mirror planet composed of $100 \%$ mirror matter with zero photon-mirror photon kinetic mixing. In fact even if photon-mirror photon mixing is non-zero, then a pure mirror planet would still be (to an extremely good approximation) transparent (with zero albedo) [20]. However, such an idealized system would not be expected to exist. Even if there was negligible amount of ordinary matter in the mirror planet when it was formed, the mirror planet will accrete ordinary matter from the host star due to that star's solar wind, and also from comets, asteroids and cosmic rays. Using the sun's solar wind as a concrete example, the current mass loss of the sun due to the solar wind is estimated to be (see e.g. Ref. [21])

$$
\frac{d M_{\odot}}{d t} \approx 3 \times 10^{-14} M_{\odot} / \text { year }
$$

This implies an accretion rate of ordinary matter onto the mirror planet of roughly,

$$
\frac{d M}{d t} \approx \frac{R_{p}^{2}}{4 r_{p}^{2}} \frac{d M_{\odot}}{d t} \sim 10^{-2} M_{J}\left(\frac{R_{p}^{2}}{r_{p}^{2}}\right) / G y r,
$$

where $R_{p}$ is the effective radius of the ordinary matter in the mirror planet, $r_{p}$ is the distance of the planet from the host star and $M_{J}$ is the mass of Jupiter. Let us now estimate $R_{p}$ by assuming hydrostatic equilibrium, which should be valid. Denoting the density of ordinary matter in the planet by $\rho^{(o)}$ and the (assumed) much larger density of mirror matter by $\rho^{(m)}$ then the condition for hydrostatic equilibrium is that the pressure $(\mathrm{P})$ gradient balances the force due to gravity, i.e.

$\S$ For the current experimental status of the mirror world solution to the solar and atmospheric neutrino anomalies, see Ref. [13] and Ref. [14] respectively. 


$$
\frac{d P}{d r}=-\rho^{(o)} g
$$

where $g$ is the local acceleration due to gravity at a distance $r$ from the center of the planet. Assuming that $\rho^{(m)} \gg \rho^{(o)}$ and taking $\rho^{(m)}$ approximately constant (i.e. independent of $r$ ) then $g$ is simply

$$
g \approx \frac{4 \pi G \rho^{(m)} r}{3}
$$

Of course this is only valid for $r<R_{m}$ where $R_{m}$ is the mirror matter radius ${ }^{\text {** }}$.

We now need to relate the pressure of the ordinary matter, $P$, to its density, $\rho^{(o)}$. First, we assume that the ordinary matter is mainly molecular hydrogen, $H_{2}$ (which is quite natural if most of it arises due to accretion from the stellar wind from the host star) it. Second, the ordinary matter inside the mirror planet should be hot because it is heated at its surface by the radiation from the host star. Finally the ordinary matter doesn't feel the pressure from the surrounding mirror matter. Because it is hot, low in density and pressure, the ordinary matter should be a gas approximately obeying the ideal gas law:

$$
P=\frac{\rho^{(o)} k T}{2 m_{p}}
$$

where $k$ is Boltzmans constant, and $2 m_{p}$ is the molecular hydrogen mass. Substituting Eq.(5) and Eq.(4) into Eq.(3) and solving the resulting differential equation, we obtain the solution:

$$
\frac{\rho^{(o)}(r)}{\rho^{(o)}(0)}=\frac{T(0)}{T(r)} e^{-r^{2} / R_{x}^{2}} \text { for } r<R_{m}
$$

where

$$
R_{x} \equiv \sqrt{\frac{3 k}{4 \pi m_{p} G \rho^{(m)} \lambda}}
$$

and

$$
\lambda \equiv \frac{1}{r^{2}} \int_{0}^{r} \frac{1}{T\left(r^{\prime}\right)} d r^{2}
$$

Note that $R_{x}$ depends on $r$ through the dependence of $\lambda$ on $r$. Unfortunately it is not so easy to obtain an accurate estimation for $\lambda$ because this requires knowledge of the Temperature profile of the ordinary matter in the planet. However a crude lower limit can be obtained

${ }^{* *}$ For $r>R_{m}, g=\frac{4 \pi G \rho^{(m)} R_{m}^{3}}{3 r^{2}}=\frac{G M_{p}}{r^{2}}$.

†† Note that for high temperatures, $T \gtrsim 3000{ }^{\circ} \mathrm{K}, H_{2}$ begins to dissociate into $H_{2}^{+}$and $e^{-}$, which will increase $P / \rho^{(o)}$ thereby increasing $R_{x}$. 
by noting that the temperature should increase as $r$ decreases. This means that $\lambda<1 / T_{s}$ (where $T_{s}$ is the "surface temperature") which allows a lower limit for $R_{x}$ of

$$
R_{x} \gtrsim 5 \times 10^{3} \sqrt{\left(T_{s} / 10^{3}{ }^{o} \mathrm{~K}\right)\left(1 \mathrm{gr} / \mathrm{cm}^{3} / \rho^{(m)}\right)} \mathrm{km} .
$$

In order to estimate the (wavelength dependent) radius of the ordinary matter $\left(R_{p}\right)$ which we define as the radius within which the radiation from the host star is absorbed or scattered during a transit, we need to know the detailed chemical composition, temperature in addition to the density $\rho^{(o)}$ profile of the ordinary matter. In a recent study, Hubbard et al 22] have estimated that the pressure where the transiting planet HD209458b becomes opaque to be roughly 10 mbar which corresponds to a density of about $\rho^{(o)} \sim 10^{-7} \mathrm{gr} / \mathrm{cm}^{3}$ [from Eq.(5)]. Since our ordinary matter enriched mirror world should have a similar surface temperature (because for close-in planets the source of the energy emitted is dominated by the irradiation from the host star rather than due to the planets internal energy) to that assumed by Hubbard et al [22], which is $\sim 1500^{\circ} \mathrm{K}$, then we may expect that our mirror world should become opaque at about the same density. Thus, assuming a total mass of ordinary matter of about few $\times 10^{-4} M_{J}$ as suggested by Eq.(2) we then estimate that

$$
R_{p} \approx 4 R_{x} .
$$

Actually, because $\rho^{(o)}$ is a steeply falling distribution for $r \gtrsim R_{x}$ the above estimate of $R_{p} / R_{x}$ should be reasonably robust. For example, if we assumed that the pressure or densities, $\rho^{(m)}, \rho^{(o)}$, were an order of magnitude larger (or smaller), then our estimate of $R_{p} / R_{x}$ would change by only about $10 \%$ (although $R_{x}$ itself depends sensitively on $\rho^{(m)}$ ). Of course, if $R_{p} \approx 4 R_{x} \gtrsim R_{m}$ then it means that the distribution of ordinary matter is extended beyond the radius of the mirror matter, $R_{m}$, in which case we may expect $R_{p}$ to be somewhat larger than $4 R_{x}$ because the ordinary matter density falls off more slowly for $r>R_{m}$ due to the weaker gravity.

However, as discussed earlier, our largest source of uncertainty in $R_{p}$ derives from its dependence on $\lambda$ though $R_{x}$. The quantity $\lambda$ (which we need to evaluate at $r \approx R_{p}$ ) should be dominated by the temperature profile in the outer regions $\left(r>0.6 R_{p}\right)$ where the conditions should not be so different from the temperature profile computed for close-in giant planets made from ordinary matter. This suggests that $\lambda \sim 1 /\left(5 T_{s}\right)$ which should be accurate to within a factor of two or so. For the transiting planet HD209458b, which is the only planet for which $R_{p}, M_{p}$ have been measured, the parameters $R_{p}, M_{p}$ are [23] $R_{p}=1.40 \pm 0.17 R_{J}$ and $M_{p}=0.69 \pm 0.05 M_{J}$. Since the mass of HD209458b is roughly that of Jupiter, we can use Jupiter as a guide to the most likely size for $R_{m} \rightarrow$. This is possible because the surface temperature of Jupiter is dominated by internal energy (rather than by solar irradiation). This leads to an expected radius of $R_{m} \approx R_{J}$ 降. This implies a

\footnotetext{
¥† For ordinary large hydrogen planets, $R_{p}$ depends quite weakly on $M_{p}$ (e.g. $R_{\text {Saturn }} / R_{J} \simeq 0.84$, while $M_{\text {Saturn }} / M_{J} \simeq 0.33$ ).
}

$\S \S$ If photon-mirror photon kinetic mixing is relatively large, then $R_{m}$ can be significantly larger 
$\bar{\rho}^{(m)} \approx 1 \mathrm{gr} / \mathrm{cm}^{3}$. Thus, we estimate that the effective radius at which the transiting planet HD209458b becomes opaque to be roughly,

$$
R_{p} \approx 4 R_{x} \sim R_{J}
$$

which is consistent with the measured value given our admittedly large theoretical uncertainty. Nevertheless, our simple analysis shows that the transit of HD209458b can be plausibly explained with the mirror planet hypothesis. Furthermore we can make some rough quantitative predictions. In particular, our simple analysis predicts that

$$
R_{p} \approx 4 R_{x} \propto \sqrt{\frac{T_{s}}{\rho^{(m)}}} \propto \sqrt{\frac{T_{s}}{M_{p}}} .
$$

Of course this is only a very rough prediction, especially the dependence on $T_{s}$ which is just the surface temperature (recall it is really the more complicated function $\lambda$ that we need in order to determine $R_{x}$ and hence $R_{p}$ ). Nevertheless, heuristically it can be understood quite easily. Increasing $M_{p}$ increases the force of gravity which causes the gas of ordinary matter to become more tightly bound to the mirror planet (thereby decreasing the effective size, $R_{p}$ ), while increasing the temperature of the gas increases the volume that the gas occupies (thereby increasing $R_{p}$ ). By contrast, the size of ordinary planets (i.e. planets made mostly of ordinary matter) depends quite weakly on their mass $M_{p}$.

Thus our hypothesis that the close-in extra-solar planets may in fact be mirror worlds may help test the mirror matter model. The rough prediction, Eq.(12) can be tested as soon as another transiting close-in planet is observed (which should occur in the near future given their rate of discovery). This should provide a significant test of the mirror world hypothesis because the radius of ordinary planets depends much more weakly on the mass of the planet. For example, the planet $\tau$ Boo b has an estimated mass of about $\approx 7 M_{J}$ [24] which means that it is about 10 times heavier than HD209458b. Thus we predict its effective radius to be roughly $\sqrt{10}$ times less than the radius of HD209458b, i.e. only about $0.5 R_{J}$. On the other hand, for planets made of ordinary matter the radius of $\tau$ Boo $\mathrm{b}$ is predicted to be [25] $R_{p} \simeq 1.2 R_{J}$ which is only about $15 \%$ less than for HD209458b.

Our prediction Eq.(12) also has important implications for measurements of reflected light (albedo). In particular a reasonably stringent limit on the albedo exists for the planet $\tau$ Boo b. In Ref. [24], they obtain an upper limit on the opposition planet/star flux ratio of $\epsilon<3.5 \times 10^{-5}$ (for wavelengths between 387.4 and $586.3 \mathrm{~nm}$ ) at $99.9 \%$ C.L. Given that $\epsilon=p\left(R_{p} / r_{p}\right)^{2}$ this translates into a limit on the geometric albedo of the planet, $p$, of $p<0.22$ at $99.9 \%$ C.L, assuming a planet radius of $R_{p} \simeq 1.2 R_{J}$. In comparison, the corresponding mean geometric albedo of Jupiter is about 0.55. However, if $\tau$ Boo b is a mirror world then we expect $R_{p} \approx 0.5 R_{J}$, as discussed above. Thus in this case, the "limit" on the albedo is $p<1.3$ which is obviously no limit, since $p$ must be less than 1 . Thus, the low value of $\epsilon$ for $\tau$ Boo b is explained simply because its effective size is expected to be small in our mirror

because the mirror matter can be heated by transfer of heat from the ordinary to the mirror matter thereby preventing the mirror surface to cool. 
world interpretation. Of course, for lighter planets, their effective size will be larger, which can make their reflected light easier to detect.

In conclusion we have argued that the hypothesis that the close-in large extra solar planets are in fact mirror worlds can explain the transit of HD209458b. The mirror world is opaque because it would accrete a significant amount of ordinary matter from the solar wind from the host star, which gives the mirror planet an effective radius large enough to explain the transit observations of HD209458b. This explanation can also nicely explain the low effective albedo of $\tau$ Boo b. Importantly, the close-in mirror world hypothesis can be tested as more transits of close-in large planets are observed. Thus, we are left with the remarkable

prospect that extrasolar planetary astronomy may provide a novel means of testing whether the fundamental interactions of particle physics conserve parity invariance.

\section{Acknowledgement}

The author would like to thank Henry Lew, Sasha Ignatiev and Ray Volkas for discussions. The author is an Australian Research Fellow. 


\section{REFERENCES}

[1] For a review and references on extrasolar planets, see the extrasolar planet encyclopaedia: http://cfa-www.harvard.edu/planets/encycl.htm]; see also M. A. C. Perryman, astro-ph/0005602.

[2] D. Charbonneau et al, Ap J. 529, L15 (2000); G. W. Henry et al, Ap J. 529, L41 (2000).

[3] R. Foot, Phys. Lett. B471, 191 (1999).

[4] T. D. Lee and C. N. Yang, Phys. Rev. 104, 256 (1956); I. Kobzarev, L. Okun and I. Pomeranchuk, Sov. J. Nucl. Phys. 3, 837 (1966); M. Pavsic, Int. J. Theor. Phys. 9, 229 (1974).

[5] R. Foot, H. Lew and R. R. Volkas, Phys. Lett. B272, 67 (1991).

[6] S. Barr, D. Chang and G. Senjanovic, Phys. Rev. Lett. 67, 2765 (1991); R. Foot and H. Lew, hep-ph/9411390; Z. G. Berezhiani and R. N. Mohapatra, Phys. Rev. D52, 6607 (1995); R. Foot, H. Lew and R. R. Volkas, JHEP 0007, 032 (2000).

[7] S. L. Glashow, Phys. Lett. B167, 35 (1986).

[8] B. Holdom, Phys. Lett. B166, 196 (1985).

[9] R. Foot, H. Lew and R. R. Volkas, Mod. Phys. Lett. A7, 2567 (1992).

[10] S. I. Blinnikov and M. Yu. Khlopov, Sov. J. Nucl. Phys. 36, 472 (1982); Sov. Astron. 27, 371 (1983); E. W. Kolb, M. Seckel and M. S. Turner, Nature 514, 415 (1985); M. Yu. Khlopov et al, Soviet Astronomy, 35, 21 (1991); M. Hodges Phys. Rev. D47, 456 (1993); Z. G. Berezhiani, A. Dolgov and R. N. Mohapatra, Phys. Lett. B375, 26 (1996); Z. G. Berezhiani, Acta Phys. Polon. B27, 1503 (1996); G. Matsas et al., hepph/9810456; Z. Silagadze, Mod. Phys. Lett. A14, 2321 (1999); hep-ph/0002255; N. F. Bell and R. R. Volkas, Phys. Rev. D59, 107301 (1999); S. I. Blinnikov, hep-ph/9902305; astro-ph/9911138; R. R. Volkas and Y. Y. Y. Wong, Astropart. Phys. 13, 21 (2000); V. Berezinsky and A. Vilenkin, hep-ph/9908257; A. Yu. Ignatiev and R. R. Volkas, Phys. Rev. D62, 023508 (2000); Phys. Lett. B487, 294 (2000); N. F. Bell, Phys. Lett. B479, 257 (2000); R. M. Crocker, F. Melia and R. R. Volkas, astro-ph/9911292; Z. Berezhiani, D. Comelli and F. L. Villante, hep-ph/0008105.

[11] Z. Silagadze, Phys. At. Nucl. 60, 272 (1997); S. Blinnikov, astro-ph/9801015; R. Foot, Phys. Lett. B452, 83 (1999); R. Mohapatra and V. Teplitz, astro-ph/9902085.

[12] R. Foot, H. Lew and R. R. Volkas, Mod. Phys. Lett. A7, 2567 (1992); R. Foot, Mod. Phys. Lett. A9, 169 (1994); R. Foot and R. R. Volkas, Phys. Rev. D52, 6595 (1995).

[13] R. Foot, Phys. Lett. B483, 151 (2000) and references there-in.

[14] R. Foot, Phys. Lett. B496, 169 (2000) and references there-in.

[15] R. Foot and R. R. Volkas, Phys. Rev. D61, 043507 (2000); Astroparticle Phys. 7, 283 (1997).

[16] R. Foot and S. N. Gninenko, Phys. Lett. B480, 171 (2000).

[17] S. I. Blinnikov and M. Yu. Khlopov, Sov. J. Nucl. Phys. 36, 472 (1982).

[18] M. R. Zapatero Osorio et al., Science 290, 103 (2000). See also M. Tamura et al., Science 282, 1095 (1998); P. W. Lucas and P. F. Roche, Mon. Not. R. Astron. Soc. 314, 858 (2000).

[19] R. Foot, A. Yu. Ignatiev and R. R. Volkas, astro-ph/0010502.

[20] R. Foot, A. Yu. Ignatiev and R. R. Volkas, astro-ph/0011156.

[21] Modern Astrophysics, by B. W. Carroll and D. A. Ostlie (Addison-Wesley, 1996). 
[22] W. B. Hubbard et al, astro-ph/0101024.

[23] T. Mazeh et al, Ap J, 532, 55 (2000).

[24] A. Cameron et al, astro-ph/0012186; See also D. Charbonneau and R. W. Noyes, astro$\mathrm{ph} / 0002489$.

[25] A. Burrows et al, ApJ, 534, L97 (2000). 